\title{
${ }^{18}$ F-labeled-Bioorthogonal Liposomes for In Vivo Targeting
}

\author{
Fabien Emmetiere ${ }^{1}$, Christopher Irwin ${ }^{1}$, Nerissa Therese Viola-Villegas ${ }^{1}$, Valerie Longo ${ }^{2}$, \\ Sarah M. Cheal ${ }^{1}$, Pat Zanzonico ${ }^{2}$, NagaVaraKishore Pillarsetty ${ }^{1}$, Wolfgang A. Weber ${ }^{3,4}$, \\ Jason S. Lewis ${ }^{1,4}$, and Thomas Reiner ${ }^{1,{ }^{*}}$ \\ ${ }^{1}$ Radiochemistry and Imaging Sciences Service, Department of Radiology, Memorial Sloan- \\ Kettering Cancer Center, New York, NY, 10065, USA \\ ${ }^{2}$ Department of Medical Physics, Memorial Sloan-Kettering Cancer Center, New York, NY, \\ 10065, USA \\ ${ }^{3}$ Molecular Imaging and Therapy Service, Department of Radiology, Memorial Sloan-Kettering \\ Cancer Center, New York, NY, 10065, USA \\ ${ }^{4}$ Molecular Pharmacology and Chemistry Program, Memorial Sloan-Kettering Cancer Center, \\ New York, NY, 10065, USA
}

\begin{abstract}
Liposomes are attractive vehicles for the controlled release of drugs and cytotoxins and have a long-standing history in medical research and clinical practice. In addition to established therapeutic indications, liposomes have several favorable properties for molecular imaging, including high stability and the ability to be labeled with radioisotopes as well as paramagnetic and fluorescent contrast agents. However, long circulation times and difficulties in creating targeted liposomes have proven challenges for imaging. In this study, we have addressed these limitations using a recently developed strategy for bioorthogonal conjugation, the reaction between tetrazines and trans-cyclooctenes. By coating radiolabeled liposomes with transcyclooctene and pretargeting with a tetrazine coupled to a targeted peptide, we were able to selectively enhance the retention of liposomes and bind them to tumor tissue in live animals. The rapid reaction between tetrazines and trans-cyclooctenes allowed imaging to be performed with the short-lived PET tracer ${ }^{18} \mathrm{~F}$, yielding signal-to-background activity ratios of 7:1. The covalent, bioorthogonally-driven tumor-targeting of liposomes by in vivo click chemistry is promising and should be explored for more selective and rapid delivery of radiodiagnostics and radiotherapeutics, two classes of drugs which particularly benefit from fast clearance, low non-specific binding, and the associated reduced toxicity to kidneys and bone marrow.
\end{abstract}

\section{Introduction}

High modularity, synthetic control, and favorable biological and pharmacokinetic properties have made liposomes attractive systems for drug delivery in recent years. ${ }^{1}$ In general, liposomal compositions achieve enrichment in areas of interest through one or a combination of two independent mechanisms: either non-specific increased retention and

\footnotetext{
*Corresponding author: T. Reiner, PhD, Department of Radiology, Memorial Sloan-Kettering Cancer Center, 1275 York Avenue, New York, NY 10065, reinert@mskcc.org; Phone: +1 646888 3461;.

Associated Content

Supporting Information

Experimental synthetic details of cold and hot precursors and liposomes; Description of biological experiments; Additional supplemental figures. This information is available free of charge via the Internet at http://pubs.acs.org/.

The authors declare no competing financial interest.
} 
accumulation in tumors due to irregular vascular architecture, abnormal fluid transport dynamics and highly porous blood vessels (enhanced permeability and retention, EPR) $)^{2-4}$ or the coating of liposome surfaces with biologically active materials selective to tumor tissues. ${ }^{1,5}$ The successful use of liposomes as drug delivery agents is reflected in the amount of effort devoted to liposome-based imaging agents and radiotherapeutics. ${ }^{6}$ In particular, the combination of PET imaging agents and liposomal nanomaterials has been the focus of numerous successful research projects in recent years. Although conceptionally attractive, both EPR-driven as well as targeted liposomal imaging agents can demonstrate a low signal/ noise ratio and a slow accumulation of the liposomes.

In this study, we have aimed to enhance the EPR-effect by capitalizing on bioorthogonal functional groups, which chemically bind and covalently immobilize the liposomes in vivo. Over the last few years, bioorthogonally reactive small molecules have attracted attention as chemical and covalent binding partners for targets of interest in vivo, enhancing the specificity of biomolecular vectors. One of the fastest chemically orthogonal reactions is the tetrazine/trans-cyclooctene inverse electron demand Diels-Alder cycloaddition (IEDDA). ${ }^{7}$ This reaction found widespread application as a tool for the in vitro and in vivo labeling of biologically relevant probes and has the potential to have a transformational impact on the development of novel radiotracers for diagnostic and radiotherapeutic applications. ${ }^{8}$ More importantly, multiple studies have shown that the reaction of tetrazines and transcyclooctenes is not only fast but that the two small molecules are also stable enough for the bioorthogonal click-reaction to occur in vivo on the cell surface in tumor xenografts. ${ }^{9-12}$

Moving beyond currently available technologies for the design of liposomes, we hypothesized that the bioorthogonal IEDDA cycloaddition between tetrazine and transcyclooctene might offer a near-ideal solution to the biggest pitfall of liposome-imaging with radioisotopes: slow accumulation in the target. We hypothesized that bioorthogonally reactive trans-cyclooctenes on the surface of liposomes will enable them to selectively accumulate in tetrazine-seeded tissues. Further, we contend that the use of bioorthogonally reactive groups enhances the intrinsic EPR effect and that such bioorthogonal liposomes are able to target specific molecular or functional characteristics of cancer cells, offering maximal versatility, modularity, and applicability. Here, we report on the synthesis, development and biological validation of such bioorthogonally functionalized liposomes. The liposomes were labeled with ${ }^{18} \mathrm{~F}$, and their uptake was measured in tetrazine-decorated tumors versus non-tetrazine tumors.

\section{Results and Discussion}

The conceptual methodology for the construction of such bioorthogonally labeled ${ }^{18} \mathrm{~F}$ liposomes is illustrated in Figure 1. ${ }^{18} \mathrm{~F}$-labeling allows their detection as well as the quantitative determination of their distribution over time by PET. In addition to the radiotracer, the liposome surface is decorated with trans-cyclooctene molecules, enabling the liposome to undergo bioorthogonal reactions. For the individual components of the liposome, we chose DSPC as a primary lipid, together with cholesterol to increase biological stability. We added a PEG-modified (2 kDa) DSPE lipid to the liposome formulation to increase the blood half-life in a manner analogous to an approach previously described. ${ }^{13}$ Further, the bioorthogonal reactivity was provided by PEG-labeled DSPE lipids which were capped with trans-cyclooctene. To these materials (DSPC:Cholesterol:DSPE-PEG2k:DSPEPEG2k-TCO $=26: 12: 1: 1$, we added ${ }^{18}$ F-labeled dipalmitoyl glycol as the radioactive tracer. In subsequent in vivo experiments, the blood/activity concentration of the resulting liposome drops by less than $20 \%$ over 5.5 hours time, indicating a long biological half-life of the ${ }^{18} \mathrm{~F}$-labeled and bioorthogonally reactive nanomaterial. 
In order to illustrate that the behavior of the liposomes can be altered from non-targeted blood-pool circulation to targeted accumulation in tumor tissues, we chose to selectively mark tumor tissue using the $\mathrm{pH}$ (Low) Insertion Peptide (pHLIP). ${ }^{14-16}$ The 37 amino acid peptide pHLIP exploits a common byproduct of the modified metabolic pathways and cellular machinery in malignant tissue: an increase in extracellular acidity. ${ }^{17-19}$ The linear pHLIP peptide was designed to change its conformation in an acidic environment. More specifically, it adopts an a-helical conformation exclusively in low-pH medium, whereupon it can translocate itself across the lipid bilayer of a cell (Fig. 2). We reasoned that a tetrazine conjugated pHLIP (pHLIP-Tz) would enable us to selectively label the surface of tumor cells with the bioorthogonal tetrazine tag (Fig. 2). After injection of the ${ }^{18} \mathrm{~F}$-liposome, the bioorthogonal nanoparticle will be able to react with the pHLIP-Tz, resulting in its covalent immobilization on the cells. This will result in accumulation of radioactivity in tumor tissues, which then can be visualized and quantified using PET.

The pHLIP-Tz was generated similarly to reaction schemes utilized by us previously (Fig. 3). ${ }^{20} \mathrm{We}$ based it on a tetrazine-precursor with a carboxylic acid functional group (Tz$\mathrm{COOH}$, Yang et al., 2012 $)^{21}$ which we reacted in the presence of benzotriazole-1-yl-oxytris-(dimethylamino)-phosphonium hexafluorophosphate (BOP) and triethylamine with an amine-functionalized maleimide to yield a tetrazine/cysteine crosslinker, maleimide-Tz (Fig. 3A). Figure 3B shows HPLC and ESI-MS chromatograms of the pure product. To yield the final bioorthogonal biomolecule, maleimide-Tz was used to derivatize a 37 amino acid pHLIP-peptide. The peptide is based on wild-type (WT) pHLIP, ${ }^{14-16}$ and its extracellular sequence includes a cysteine, which we used to covalently react with maleimide-Tz in PBS at room temperature. After HPLC purification, we were able to obtain the bioorthogonally reactive pHLIP-Tz in $42 \%$ yield and greater than $98 \%$ purity, as shown by HPLC and LCMS analysis (Fig. 3C-3D). In order to measure the stability of tetrazine functional groups in vivo towards decomposition in biological media, we dissolved 3-(4-phenylacetic acid)-1,2,4,5-tetrazine (Tz-COOH) in PBS and serum. The half-life of this tetrazine-label, estimated by HPLC in the presence of an internal standard, was measured to be $73.4 \pm 1.6 \mathrm{~h}$ and $28.5 \pm 0.1 \mathrm{~h}$, respectively, in PBS and human serum (Fig. S1), similar to what has been found before. ${ }^{22}$ Although the decomposition rates are increased in whole blood, a half-life in the order of hours is sufficient for pretargeting and in vivo click chemistry. ${ }^{9-12}$

For the synthesis of the trans-cyclooctene conjugate, we chose the PEGylated and amineterminated DSPE-PEG2k as the lipid starting material. We reacted the $(E)$-cyclooct-4-enyl 2,5-dioxo-1-pyrrolidinyl carbonate (TCO-NHS) in dichloromethane with the lipid to yield the resulting DSPE-PEG2k-TCO in 47\% isolated yield (Fig. 4A). After the reaction and subsequent silica gel purification, mass spectra analysis (ESI-MS) showed that no lipid starting material was present in the reaction mixture, indicating successful conversion (Fig. 4B). $\left[{ }^{18} \mathrm{~F}\right]-\mathrm{FDP}$ was produced based on a synthetic procedure published earlier. ${ }^{13}$ The radiolabeled lipid was obtained by incorporation of ${ }^{18} \mathrm{~F}$ into the tosylated starting material (DP-OTs) via nucleophilic substitution, yielding the purified product after Sep-Pak purification in quantities of $21 \mathrm{mCi} \pm 5 \mathrm{mCi}$ and decay-corrected radiochemical yields (dcRCY) of $41 \% \pm 5 \%$ (Fig. $4 \mathrm{C}$ ). The radiochemical purity was measured to be $\geq 96 \%$ by iTLC (Fig. 4D).

Together with DSPE-PEG2k-TCO, DSPE-PEG2k, DSPC and cholesterol, $\left[{ }^{18} \mathrm{~F}\right]-\mathrm{FDP}$ was used for the assembly of ${ }^{18} \mathrm{~F}$-TCO-liposomes via sonication at $55^{\circ} \mathrm{C}$ (Fig. 5A). Specifically, we added the radiolabeled $\left[{ }^{18} \mathrm{~F}\right]$-FDP, dissolved in DCM, to a previously prepared mixture of the other liposome-components. The organic solvent was then removed, PBS added and the mixture sonicated for 30 minutes. The resulting heterogeneous dispersion was then filtered using $220 \mathrm{~nm}$ size exclusion filters, yielding the radiolabeled bioorthogonal ${ }^{18} \mathrm{~F}$ TCO-liposomes in quantities of $3 \mathrm{mCi} \pm 2 \mathrm{mCi}(\mathrm{RCP}>95 \%$, dcRCY: $15 \% \pm 4 \%, \mathrm{n}=5)$. 
Dynamic light scattering showed that the ${ }^{18} \mathrm{~F}$-TCO-liposomes have comparable sizes and PdIs $(d=142.9 \mathrm{~nm}, \mathrm{PdI}=0.207$, Fig. 4D) to their cold non-labeled counterparts $(\mathrm{d}=130.3$ $\mathrm{nm}, \mathrm{PdI}=0.081$, Fig. S2). Likewise, we found that after sonication and purification, the liposomal preparation contains exclusively liposomes $\left(t_{R}=19.3 \mathrm{~min}\right.$, NMW $>670 \mathrm{kDa}$, Fig. 4E) and no free $\left[{ }^{18} \mathrm{~F}\right]-\mathrm{FDP}\left(\mathrm{t}_{\mathrm{R}}=11.8 \mathrm{~min}, \mathrm{NMW}<2 \mathrm{kDa}\right.$, Fig. S2). To elucidate whether the liposome formation results in degradation or rearrangement of the trans-cyclooctene, two solutions of DSPE-PEG2k-TCO in DCM were prepared, one of which was subjected to sonication at $55{ }^{\circ} \mathrm{C}$. No difference in reactivity between the sonicated and the non-sonicated solution with $\mathrm{Tz}-\mathrm{COOH}$ was observed, indicating that the trans-cyclooctenes retain their reactivity after the ${ }^{18}$ F-TCO-liposome formation.

For subsequent in vivo experiments - and as a proof of principle for this concept - tumorbearing athymic nude mice $(n=3)$ were obtained by injection of SKOV3 ovarian cancer cells $\left(5 \times 10^{6}\right.$ cells in 1:1 PBS:BD Matrigel) into both shoulders, and the tumors were allowed to grow and vascularize for 2 weeks. Before injection of the PET active ${ }^{18} \mathrm{~F}-\mathrm{TCO}$ liposomes, pHLIP-Tz $(50 \mu \mathrm{L}, 0.89 \mathrm{mM})$ was administered intratumorally into one of the tumors (Fig. 4A-4B). The pHLIP-Tz was allowed to extravasate into the tumor tissue (10 min incubation time), before ${ }^{18} \mathrm{~F}$-TCO-liposomes $(\sim 250 \mu \mathrm{Ci}, 200 \mu \mathrm{L}$ PBS $)$ were injected intravenously. As anticipated, the liposomes show a long blood half-life, with a large portion of the ${ }^{18} \mathrm{~F}$-probe still remaining in the bloodstream after 2 hours. A portion of the liposome is excreted via the hepatobiliary system, which can be a combination of pHLIP-Tz binding in these tissues and partial excretion of the liposome. ${ }^{23}$ At 1 hour, $12.8 \pm 2.0 \% \mathrm{ID} / \mathrm{g}$ was found in the liver and $16.0 \pm 8.0 \% \mathrm{ID} / \mathrm{g}$ in the intestines, whereas the kidneys did not show any significant accumulation. The ${ }^{18}$ F-liposome is not stable to defluorination, with $2.7 \pm$ $1.3 \% \mathrm{ID} / \mathrm{g}$ of the liposome accumulating in the bone after 60 minutes. Immediately after injection, the liposomes begin to accumulate in the SKOV3 tumor tissue marked with pHLIP-Tz, resulting in the tumors being visible by PET as early as 30 minutes. After 120 minutes, we achieved an activity concentration of $3.5 \pm 1.2 \% \mathrm{ID} / \mathrm{g}$ in the tumors marked with pHLIP-Tz, as opposed to $0.46 \pm 0.04 \% \mathrm{ID} / \mathrm{g}$ for the tumors without pHLIP-Tz. This was also reflected in the tumor/muscle ratios, which were $6.9 \pm 1.4$ for the SKOV3 tumors marked with pHLIP-Tz and $0.95 \pm 0.19$ for unmarked SKOV3 tumors (Fig. 4C-4D). Therefore, owing to the rapid reaction rate of tetrazines and trans-cyclooctenes, we were able to increase the uptake of the liposome in tissues of interest to levels greater than that achieved by the more commonly exploited EPR-effect alone.

In order to ensure that the observed preferential accumulation in tetrazine-enriched tumors is based on the bioorthogonal IEDDA reaction and not disruption of the vasculature, experiments were conducted with and without the injection of vehicle (50 $\mu \mathrm{L}, \mathrm{PBS})$ in the control tumor. In both scenarios, the uptake of ${ }^{18} \mathrm{~F}$-TCO-liposomes was significantly smaller than in the pHLIP-Tz positive tumor $(0.46 \pm 0.27 \% \mathrm{ID} / \mathrm{g}$ without and $0.41 \pm 0.37 \% \mathrm{ID} / \mathrm{g}$ with vehicle-injection, both at $60 \mathrm{~min}$ ). No statistically significant difference between muscle tissue and unmarked tumors was observed. Likewise, when the experiment was repeated without the use of DSPE-PEG2k-TCO in the liposome formulation (yielding nonbioorthogonal ${ }^{18} \mathrm{~F}$-liposomes), we did not see a statistically significant difference between the two SKOV3-tumors $(1.15 \pm 0.69 \% \mathrm{ID} / \mathrm{g}$ for the SKOV3 tumor marked with pHLIP-Tz and $1.10 \pm 0.64 \% \mathrm{I} \mathrm{D/g}$ for the tumor injected with vehicle, both at $\mathrm{t}=60 \mathrm{~min})$. The ${ }^{18} \mathrm{~F}$ TCO-liposomes remain bioorthogonally reactive even after long circulation times. When, 5.5 hours after intravenous ${ }^{18} \mathrm{~F}$-TCO-liposome injection, one of the SKOV3-tumors is marked with an injection of pHLIP-Tz, the uptake of the already circulating ${ }^{18} \mathrm{~F}-\mathrm{TCO}-$ liposome in the tumor increases rapidly from $0.36 \pm 0.22 \% \mathrm{ID} / \mathrm{g}$ to $1.23 \pm 0.32 \% \mathrm{ID} / \mathrm{g}$ within 60 minutes. 
In summary, we have shown that bifunctional, bioorthogonal ${ }^{18} \mathrm{~F}$-liposomes can be designed using tetrazine/trans-cyclooctene technology and that these liposomes and their tetrazinelabeled counterparts are stable enough for in vivo applications. Further, we have found that tetrazine-labeled tumors show significantly increased uptake of radiolabeled bioorthogonal liposomes compared to the untreated tumors and that the uptake behavior and pharmacokinetics of large nanoparticulate liposomes can be altered by means of chemical modifications. This is possible because of the extremely fast reaction kinetics and high selectivity of the IEDDA reaction.

The bioorthogonal ${ }^{18} \mathrm{~F}$-liposome was developed rapidly from its lipid-based components after the introduction of the ${ }^{18} \mathrm{~F}$ radiolabel, providing maximum modularity for subsequent modifications. Liposome formulations with different compositions and reporters could also be developed and applied in similar ways. However, we believe that the described liposomes might be particularly useful for the kinetically enhanced and excretory modulated delivery of drugs, imaging agents, and nuclear therapeutics, immobilized either on the liposomal surface or in the endoliposomal space. The technology can be readily expanded to other drugs, biomolecules, and molecular targets and could facilitate the development and delivery of drugs and imaging agents.

\section{Supplementary Material}

Refer to Web version on PubMed Central for supplementary material.

\section{Acknowledgments}

The authors thank the Small Animal Imaging Core as well as the Radiochemistry and Molecular Imaging Probes Core for support. The authors thank Yana K. Reshetnyak, Oleg A. Andreev, Willem Mulder and Diane S. Abou for helpful discussions as well as Carlos Pérez-Medina for critical revision of the manuscript. Finally, the authors thank the NIH (R01 CA138468 for JSL and K25 EB016673 for TR), the Brain Tumor Center of Memorial SloanKettering Cancer (for TR) as well as the Nanotechnology Center of Memorial Sloan-Kettering Cancer Center (for $\mathrm{TR})$ for their generous funding.

\section{Abbreviations}

$\begin{array}{ll}\text { BOP } & \begin{array}{l}\text { Benzotriazole-1-yl-oxy-tris-(dimethylamino)-phosphonium } \\ \text { hexafluorophosphate } \\ \text { dichloromethane }\end{array} \\ \text { DCM } & \text { 3-tosyl-1,2-dipalmitoyl glycerol } \\ \text { DP-OTs } & \text { 1,2-distearoyl-sn-glycero-3-phosphocholine } \\ \text { DSPC } & \text { 1,2-distearoyl-sn-glycero-3-phosphoethanolamine-N- } \\ \text { DSPE-PEG2k } & \text { Enhino(polyethyleneglycol)-2000] } \\ \text { EPR } & \text { Electrospray ionization mass spectrometry } \\ \text { ESI-MS } & \text { Triethylamine } \\ \text { Et } \mathbf{3} & \text { 3-[ }{ }^{18} \text { F]-fluorodiplamitoyl-1,2-glycerol } \\ \left.{ }^{\mathbf{1 8}} \mathbf{F}\right]- \text { FDP } & \text { High-performance liquid chromatography } \\ \text { HPLC } & \text { Inverse electron-demand Diels-Alder } \\ \text { IEDDA } & \text { Instante thin layer chromatography } \\ \text { iTLC } & \end{array}$




$\begin{array}{ll}\text { NHS } & N \text {-hydroxysuccinimide } \\ \text { PBS } & \text { phosphate-buffered saline } \\ \text { PdI } & \text { Polydispersity index } \\ \text { PEG } & \text { polyethylene glycol } \\ \text { pHLIP } & \text { pH low insertion peptide } \\ \text { PET } & \text { Positron emission tomography } \\ \text { TCO } & \text { trans-cylooctene } \\ \text { Tz } & \text { tetrazine }\end{array}$

\section{References}

(1). Torchilin VP. Recent advances with liposomes as pharmaceutical carriers. Nat. Rev. Drug Discov. 2005; 4:145-60. [PubMed: 15688077]

(2). Schroeder A, Kost J, Barenholz Y. Ultrasound, liposomes, and drug delivery: principles for using ultrasound to control the release of drugs from liposomes. Chem. Phys. Lipids. 2009; 162:1-16. [PubMed: 19703435]

(3). Chauhan VP, Stylianopoulos T, Martin JD, Popović Z, Chen O, Kamoun WS, Bawendi MG, Fukumura D, Jain RK. Normalization of tumour blood vessels improves the delivery of nanomedicines in a size-dependent manner. Nature Nanotechnol. 2012; 7:383-8. [PubMed: 22484912]

(4). Matsumura Y, Maeda H. A new concept for macromolecular therapeutics in cancer chemotherapy: mechanism of tumoritropic accumulation of proteins and the antitumor agent smancs. Cancer Res. 1986; 46:6387-92. [PubMed: 2946403]

(5). Hilgenbrink AR, Low PS. Folate receptor-mediated drug targeting: From therapeutics to diagnostics. J. Pharm. Sci. 2005; 94:2135-46. [PubMed: 16136558]

(6). Petersen AL, Hansen AE, Gabizon A, Andresen TL. Liposome imaging agents in personalized medicine. Adv. Drug Deliv. Rev. 2012; 64:1417-35. [PubMed: 22982406]

(7). Blackman ML, Royzen M, Fox JM. Tetrazine ligation: fast bioconjugation based on inverseelectron-demand Diels-Alder reactivity. J. Am. Chem. Soc. 2008; 130:13518-9. [PubMed: 18798613]

(8). Devaraj NK, Weissleder R. Biomedical applications of tetrazine cycloadditions. Acc. Chem. Res. 2011; 44:816-27. [PubMed: 21627112]

(9). Rossin R, Verkerk PR, van den Bosch SM, Vulders RC, Verel I, Lub J, Robillard MS. In vivo chemistry for pretargeted tumor imaging in live mice. Angew. Chem. Int. Ed. Engl. 2010; 49:3375-8. [PubMed: 20391522]

(10). Devaraj NK, Thurber GM, Keliher EJ, Marinelli B, Weissleder R. Reactive polymer enables efficient in vivo bioorthogonal chemistry. Proc. Natl. Acad. Sci. U S A. 2012; 109:4762-7. [PubMed: 22411831]

(11). Zeglis BM, Sevak KK, Reiner T, Mohindra P, Carlin SD, Zanzonico P, Weissleder R, Lewis JS. A Pretargeted PET Imaging Strategy Based on Bioorthogonal Diels-Alder Click Chemistry. J. Nucl. Med. 2013; 54:1389-96. [PubMed: 23708196]

(12). Rossin R, Bosch S. M. v. d. Hoeve W. t. Carvelli M, Versteegen RM, Lub J, Robillard MS. Highly reactive trans-cyclooctene tags with improved stability for Diels-Alder chemistry in living systems. Bioconjug. Chem. 2013; 24:1210-1217. [PubMed: 23725393]

(13). Marik J, Tartis MS, Zhang H, Fung JY, Kheirolomoom A, Sutcliffe JL, Ferrara KW. Longcirculating liposomes radiolabeled with $\left[{ }^{18} \mathrm{~F}\right]$-fluorodipalmitin $\left(\left[{ }^{18} \mathrm{~F}\right]-\mathrm{FDP}\right)$. Nucl. Med. Biol. 2007; 34:165-71. [PubMed: 17307124] 
(14). Reshetnyak YK, Segala M, Andreev OA, Engelman DM. A monomeric membrane peptide that lives in three worlds: in solution, attached to, and inserted across lipid bilayers. Biophys. J. 2007; 93:2363-72. [PubMed: 17557792]

(15). Vavere AL, Biddlecombe GB, Spees WM, Garbow JR, Wijesinghe D, Andreev OA, Engelman DM, Reshetnyak YK, Lewis JS. A novel technology for the imaging of acidic prostate tumors by positron emission tomography. Cancer Res. 2009; 69:4510-6. [PubMed: 19417132]

(16). Segala J, Engelman DM, Reshetnyak YK, Andreev OA. Accurate analysis of tumor margins using a fluorescent pH Low Insertion Peptide (pHLIP). Int. J. Mol. Sci. 2009; 10:3478-87. [PubMed: 20111691]

(17). Gottfried E, Kreutz M, Mackensen A. Tumor metabolism as modulator of immune response and tumor progression. Semin. Cancer Biol. 2012; 22(4):335-41. [PubMed: 22414910]

(18). Vander Heiden MG, Cantley LC, Thompson CB. Understanding the Warburg effect: the metabolic requirements of cell proliferation. Sci. Signal. 2009; 324:1029.

(19). Zhang X, Lin Y, Gillies RJ. Tumor pH and its measurement. J. Nucl. Med. 2010; 51:1167-70. [PubMed: 20660380]

(20). Keliher E, Reiner T, Thurber GM, Upadhyay R, Weissleder R. Efficient ${ }^{18}$ F-Labeling of Synthetic Exendin-4 Analogues for Imaging Beta Cells. ChemistryOpen. 2012; 1:177-83. [PubMed: 23997998]

(21). Yang J, Karver MR, Li W, Sahu S, Devaraj NK. Metal-catalyzed one-pot synthesis of tetrazines directly from aliphatic nitriles and hydrazine. Angew. Chem. Int. Ed. Engl. 2012; 51:5222-5. [PubMed: 22511586]

(22). Karver MR, Weissleder R, Hilderbrand SA. Synthesis and evaluation of a series of 1,2,4,5tetrazines for bioorthogonal conjugation. Bioconjug. Chem. 2011; 22:2263-70. [PubMed: 21950520]

(23). Daumar P, Wanger-Baumann CA, Pillarsetty N, Fabrizio L, Carlin SD, Andreev OA, Reshetnyak YK, Lewis JS. Efficient ${ }^{18}$ F-labeling of large 37-amino-acid pHLIP peptide analogues and their biological evaluation. Bioconjug. Chem. 2012; 23:1557-66. [PubMed: 22784215] 

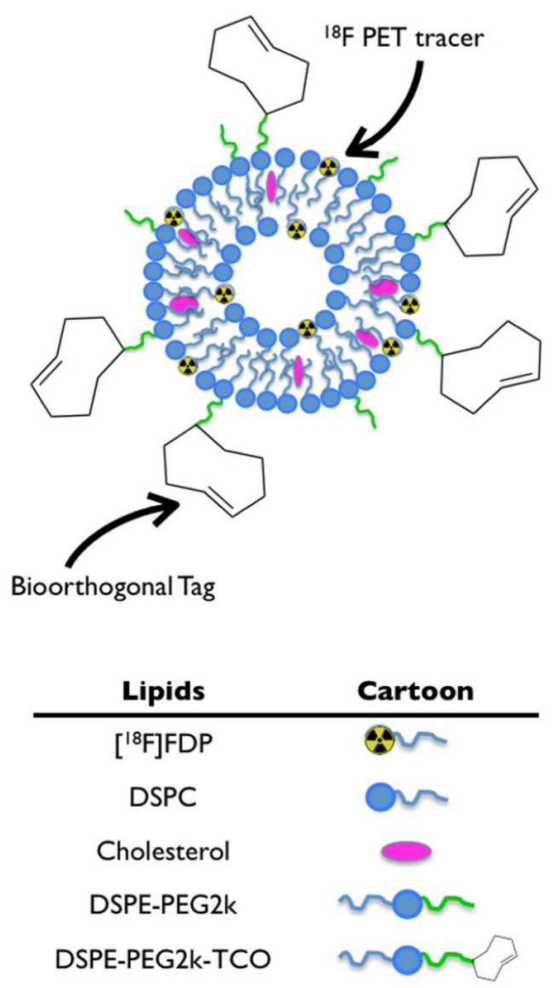

Figure 1.

Conceptual design of ${ }^{18} \mathrm{~F}$-TCO-liposomes and pHLIP-Tz coupling for pre-targeting of acidosis: Bioorthogonal ${ }^{18} \mathrm{~F}-\mathrm{TCO}$ contain DSPC and cholesterol together with the PEGylated DSPE-PEG2k for increased blood half-life. They further contain the ${ }^{18} \mathrm{~F}$-labeled dipalmitoyl $\left[{ }^{18} \mathrm{~F}\right]-F D P$ and the bioorthogonal trans-cyclooctene labeled DSPE-PEG2k-TCO, allowing them to react with tetrazine-containing tissues in vivo. 


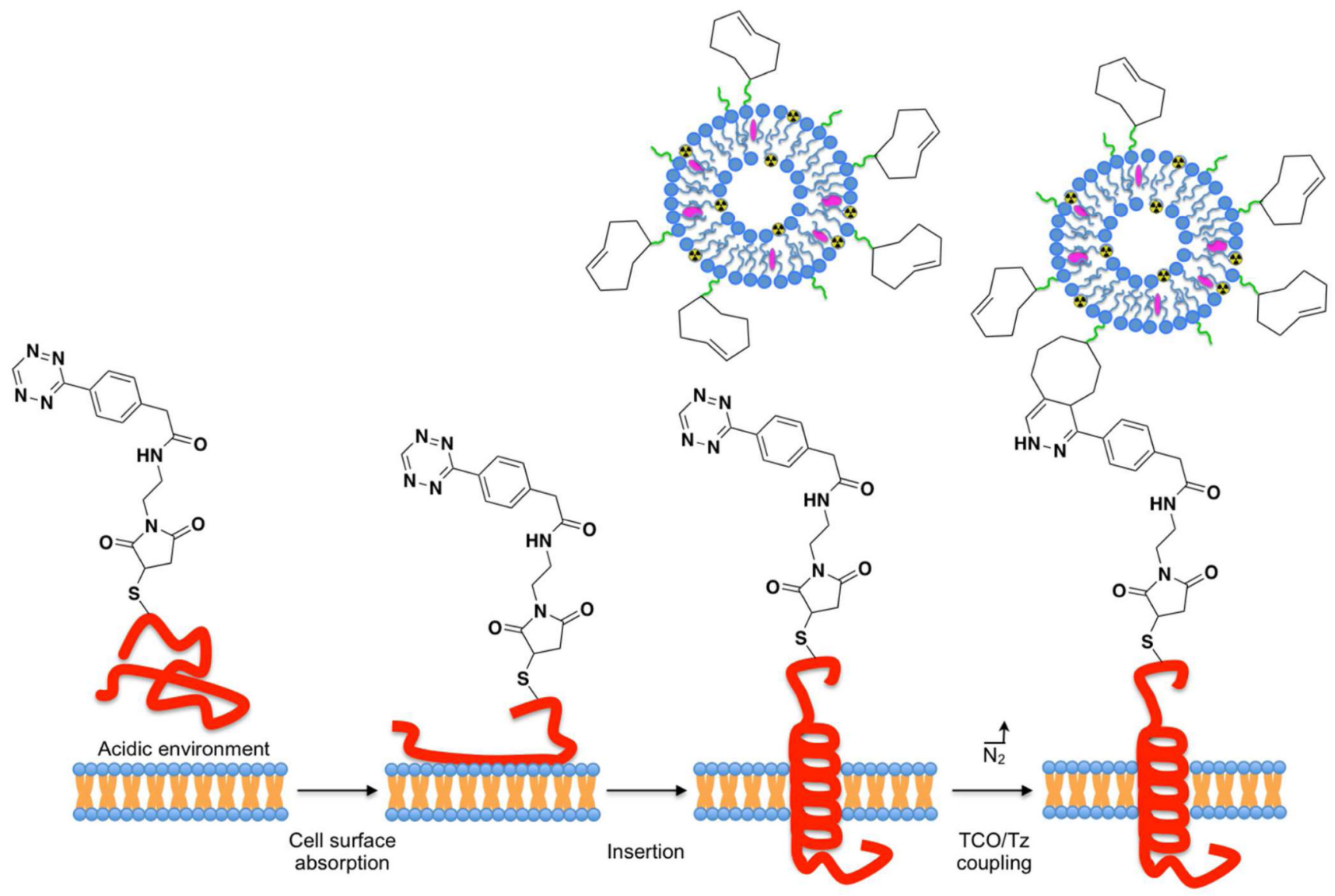

Figure 2.

Pretargeting of ${ }^{18} \mathrm{~F}$-TCO-liposomes and pHLIP-Tz rapid accumulation and covalent immobilization in tumor tissues. At low $\mathrm{pH}$ levels, the polar C-terminus of $\mathrm{pHLIP-Tz}$ changes its conformation to a helical structure, resulting in the insertion of the peptide into the cellular membrane. The resulting transmembrane helix displays a bioorthogonal tetrazine on the cell surface, which can then be targeted by bioorthogonal liposomes. Here, we designed long circulating liposomes displaying the ${ }^{18} \mathrm{~F}$-labeled lipid $\left[{ }^{18} \mathrm{~F}\right]$-FDP on the liposome surface together with the bioorthogonal trans-cyclooctene-conjugated DSPEPEG2k-TCO. Reaction of the trans-cyclooctene with pHLIP-Tz leads to covalent immobilization of the liposome on the cellular surface and subsequent enrichment of ${ }^{18} \mathrm{~F}$ in tissues with high concentrations of pHLIP-Tz. 
A<smiles>O=C(O)Cc1ccc(-c2nncnn2)cc1</smiles>

Tz-COOH

C

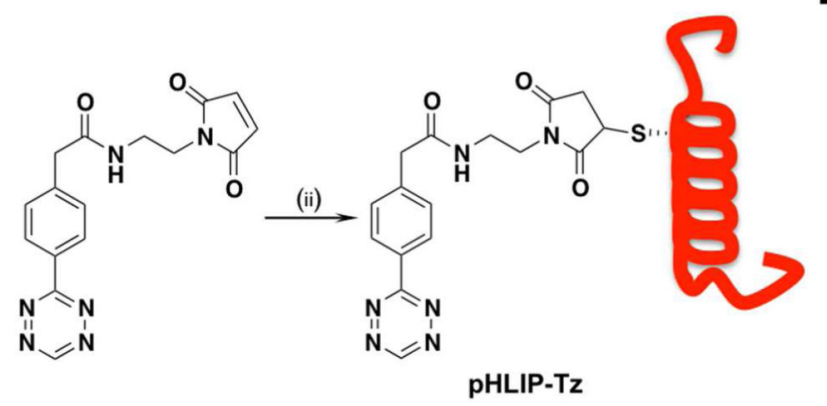

B

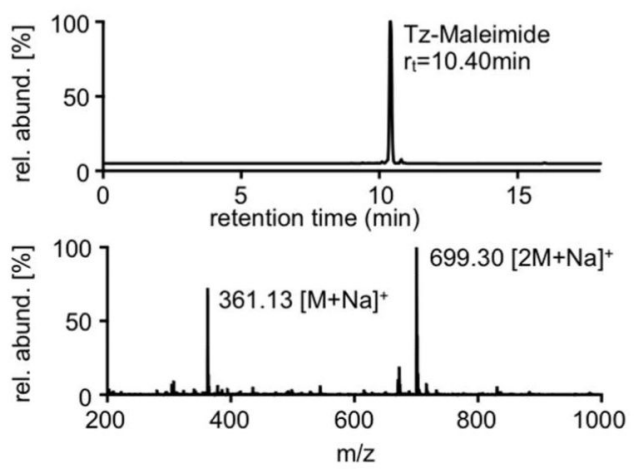

D
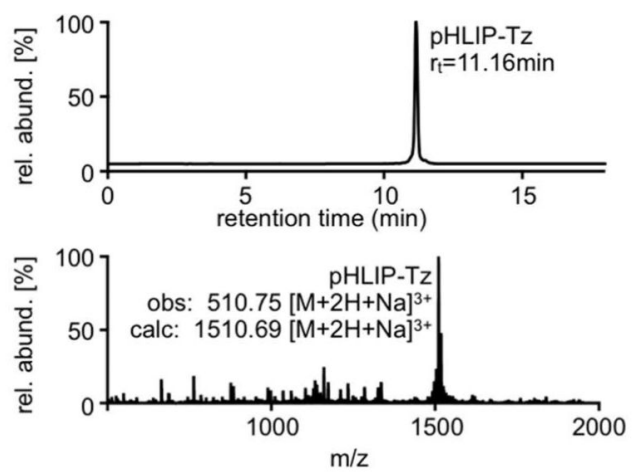

Figure 3.

Synthesis and analysis of bioorthogonal pHLIP-Tz. (A) Synthesis of Tz-maleimide;

Reagents and conditions: (i) maleic anhydride, BOP, $\mathrm{Et}_{3} \mathrm{~N}$, room temperature, $4.5 \mathrm{~h}, 34 \%$;

(B) HPLC and ESI-MS traces of Tz-maleimide; (C) Synthesis of pHLIP-Tz from Tzmaleimide Reagents and conditions: (ii) pHLIP, PBS, room temperature, 3 h, 77\%; (D)

HPLC and ESI-MS traces of pHLIP-Tz. 
A

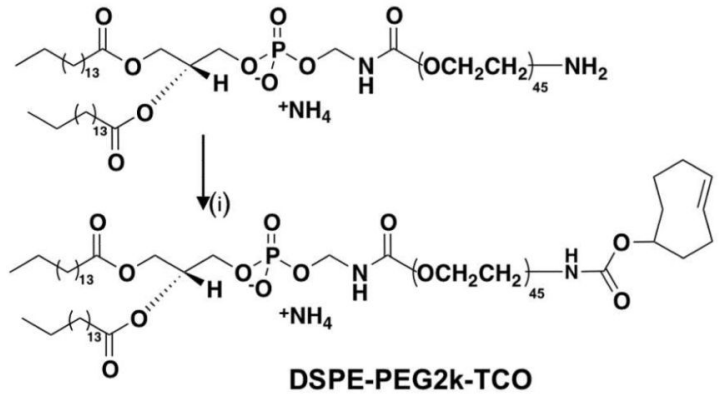

B

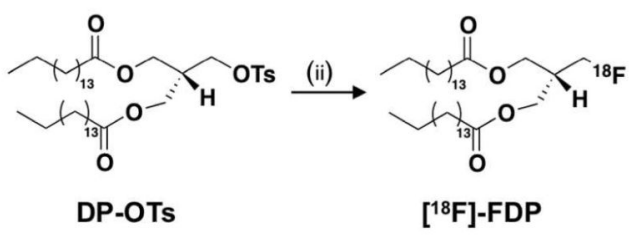

C

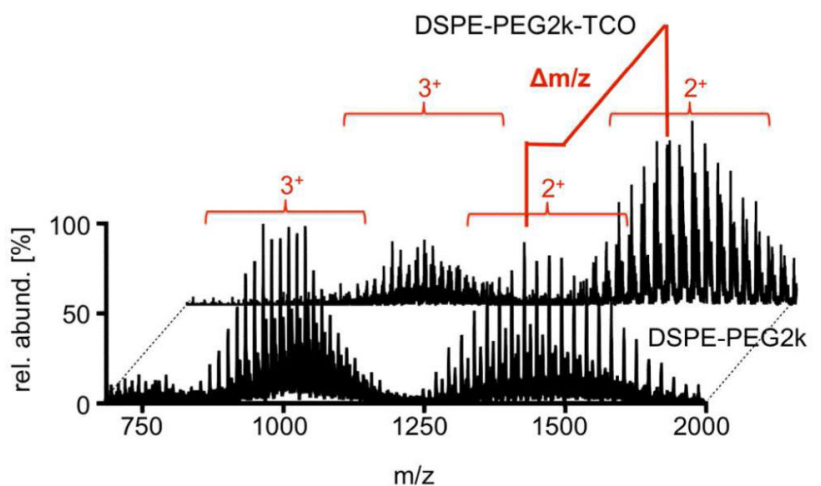

D

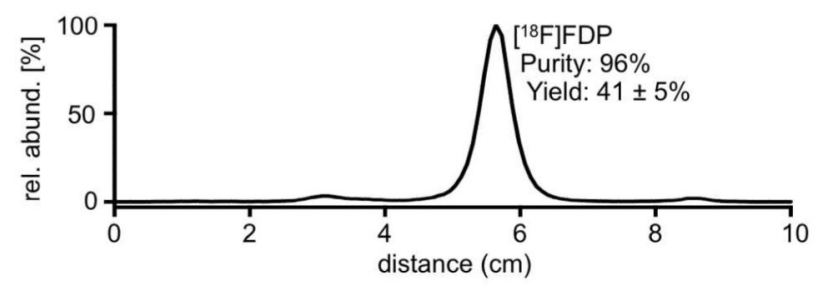

Figure 4.

Synthesis and analysis of functionalized lipid building blocks. (A) Synthesis of DSPEPEG2k; Reagents and conditions: (i) TCO-NHS, Et 3 N, DCM, room temperature, $5 \mathrm{~h}$, Yield: $47 \%$; (B) Synthesis of $\left[{ }^{18} \mathrm{~F}\right]$-FDP; Reagents and conditions: (ii) Acetonitrile, $120{ }^{\circ} \mathrm{C}, 20$ min, dcRCY: $40 \% \pm 5 \%$, RCP: 96\%; (C) ESI-MS analysis of DSPE-PEG2k-TCO versus DSPE-PEG2k; (D) iTLC of $\left[{ }^{18} \mathrm{~F}\right]-\mathrm{FDP}$; 


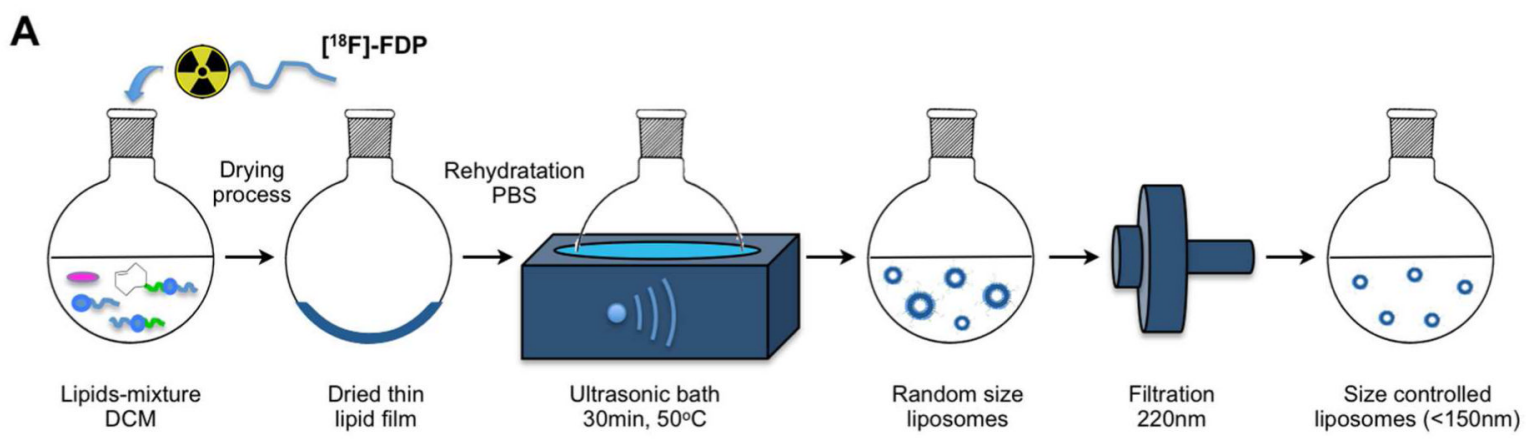

B

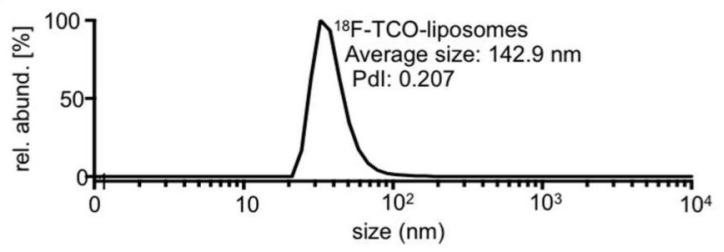

C

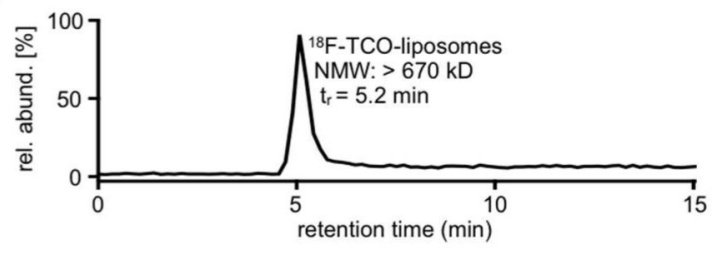

Figure 5.

Synthesis and analysis of bioorthogonal liposomes. (A) Flow scheme for the synthesis of ${ }^{18}$ F-TCO-liposomes; (B) Dynamic light scattering and (C) size exclusion chromatography of ${ }^{18} \mathrm{~F}$-TCO-liposomes. 

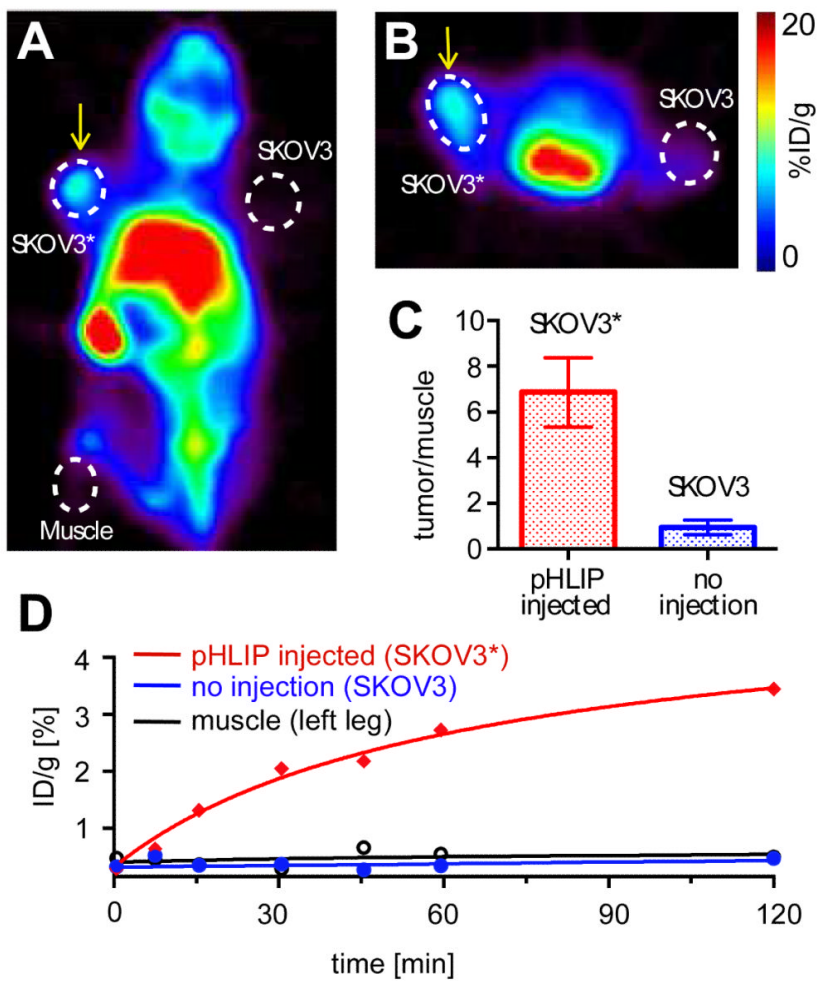

Figure 6.

Biodistribution and PET imaging of bioorthogonal ${ }^{18} \mathrm{~F}$-liposomes as a selective marker for tetrazine-labeled SKOV3 ovarian cancer xenografts. (A) PET imaging $2 \mathrm{~h}$ post-injection of ${ }^{18} \mathrm{~F}$-liposomes (intravenous, $200 \mu \mathrm{L}, 250 \mu \mathrm{Ci}$, saline) in a mouse bearing both pHLIP-Tztreated $\left(\mathrm{SKOV}^{*}\right.$, left shoulder, yellow arrow, $50 \mu \mathrm{L}, 0.89 \mathrm{mM}$, PBS) and untreated (SKOV3, right shoulder) tumors; (B) Normalized tumor/muscle ratios for treated and untreated tumor $2 \mathrm{~h}$ post-injection; (C) Time/activity curves of the tetrazine-tagged tumor, untreated tumor, and muscle (left leg). 